\title{
Etching effects during the chemical vapor deposition of (100) diamond
}

\author{
C. C. Battaile ${ }^{a)}$ \\ Sandia National Laboratories, Materials and Process Computation and Modeling, \\ Albuquerque, New Mexico 87185 \\ D. J. Srolovitz \\ University of Michigan, Department of Materials Science and Engineering, Ann Arbor, Michigan 48109 \\ I. I. Oleinik, D. G. Pettifor, and A. P. Sutton \\ University of Oxford, Department of Materials, Oxford OX1 3PH, United Kingdom \\ S. J. Harris \\ General Motors R\&D Center, Physics and Physical Chemistry Department, Warren, Michigan 48090 \\ J. E. Butler \\ Naval Research Laboratory, Gas/Surface Dynamics Section, Chemistry Division, Washington, DC 20375
}

(Received 5 April 1999; accepted 21 May 1999)

\begin{abstract}
Current theories of CVD growth on (100) diamond are unable to account for the numerous experimental observations of slow-growing, locally smooth $(100)(2 \times 1)$ films. In this paper we use quantum mechanical calculations of diamond surface thermochemistry and atomic-scale kinetic Monte Carlo simulations of deposition to investigate the efficacy of preferential etching as a mechanism that can help to reconcile this discrepancy. This etching mechanism allows for the removal of undercoordinated carbon atoms from the diamond surface. In the absence of etching, simulated growth on the $(100)(2 \times 1)$ surface is faster than growth on the (110) and (111) surfaces, and the (100) surface is atomically rough. When etching is included in the simulations, the (100) growth rates decrease to values near those observed experimentally, while the rates of growth on the other surfaces remain largely unaffected and similar to those observed experimentally. In addition, the etching mechanism promotes the growth of smooth (100) surface regions in agreement with numerous scanning probe studies. (C) 1999 American Institute of Physics.
\end{abstract}

[S0021-9606(99)70531-3]

\section{INTRODUCTION}

Despite the years of research that have already gone into understanding the chemical vapor deposition of diamond, ${ }^{1}$ the stereochemical details of diamond growth mechanisms remain unclear. Diamond film growth is usually conducted in a high-temperature hydrogen plasma in which direct monitoring of the elementary reactions responsible for growth is very difficult. Therefore, modeling of the surface chemical reactions and simulation of the growth behavior are useful tools for probing the diamond CVD process on a level of detail that is not available to direct measurement. Experiments $^{2-5}$ and modeling ${ }^{6,7}$ indicate that the most abundant active species at the diamond growth surface are atomic hydrogen $(\mathrm{H})$, methyl radicals $\left(\mathrm{CH}_{3}\right)$, and acetylene $\left(\mathrm{C}_{2} \mathrm{H}_{2}\right)$. Atomic hydrogen plays a particularly important role during diamond growth, as it is simultaneously responsible for passivating the diamond surface and activating surface sites for subsequent growth reactions. Incorporation of carbon atoms (C) into the diamond lattice occurs by the deposition of $\mathrm{CH}_{3}$ and $\mathrm{C}_{2} \mathrm{H}_{2}$ onto the surface. ${ }^{1}$

As diamond crystallites grow, they exhibit $\{100\}$ and/or $\{111\}$ facets. ${ }^{8-10}$ The $\{100\}$ surfaces are often smooth, dimer row reconstructed, and hydrogen terminated $[\{100\}(2$

a)Electronic mail: ccbatta@sandia.gov $\times 1): \mathrm{H}] .{ }^{11-13}$ The $\{110\}$ surfaces are not generally observed (except in $\{110\}$-oriented homoepitaxy ${ }^{14,15}$ ) because they grow much faster than the $\{100\}$ or $\{111\}$ faces. ${ }^{8,16}$ The rate of diamond deposition onto the $\{100\}$ and $\{111\}$ surfaces at $1200 \mathrm{~K}$ in a hot filament assisted CVD reactor is typically around $0.5 \mu \mathrm{m} / \mathrm{h},{ }^{16,17}$ and deposition onto the $\{110\}$ face occurs at around $2 \mu \mathrm{m} / \mathrm{h} .{ }^{16}$

Kinetic models ${ }^{6,18-24}$ of diamond growth have succeeded in reproducing many aspects of observed diamond growth behavior. For example, chemical kinetics modeling ${ }^{19,22}$ and atomic-scale kinetic Monte Carlo simulations, ${ }^{25}$ using diamond growth mechanisms with $\mathrm{CH}_{3}$ as the only growth species, can predict (100) growth rates that agree very well with experimental measurements. However, there are other key aspects of diamond growth behavior that current diamond growth models are unable to capture. As stated above, experiments show that the (110) surface should grow the fastest. However, the atomic-scale growth simulations ${ }^{25}$ of hot filament-assisted diamond CVD, with only methyl radical as a growth species, predict growth rates that are too slow, i.e., the (100) surface grows fastest at around $0.5 \mu \mathrm{m} / \mathrm{h}$, and the (110) and (111) growth rates are extremely low at around 0.01 and $0.001 \mu \mathrm{m} / \mathrm{h}$, respectively. This is because nucleation on the (100), (110), and (111) surfaces requires the adjacent deposition of one, two, and three $\mathrm{C}$ atoms, respectively, and the probability of realizing a deposited multicar- 
bon cluster on the diamond surface decreases rapidly as the size of the cluster increases. ${ }^{25,26}$ (The presence of surface steps in the simulations does not qualitatively change the result. $^{25}$ ) In the framework of conventional diamond growth mechanisms, ${ }^{19,20,22}$ these nucleation requirements imply that growth on the (100) surface of diamond will be fastest in any "reasonable", growth environment. ${ }^{3,7}$ Therefore, even though the predicted (110) and (111) growth rates can be brought into agreement with experiments by including both $\mathrm{CH}_{3}$ and $\mathrm{C}_{2} \mathrm{H}_{2}$ as a growth species, ${ }^{6,25,26}$ this approach produces anomalously high (100) growth rates around 2 $\mu \mathrm{m} / \mathrm{h}^{7,25}$ Furthermore, atomic-scale growth simulations ${ }^{22,25}$ predict rough (100) surfaces regardless of the choice of growth species, in contradiction to experimental observations. $^{11-13}$

These results suggest two likely scenarios. It is possible that diamond growth occurs primarily by $\mathrm{CH}_{3}$ incorporation, and that our current understanding of growth on all three surface orientations is in error. In particular, current diamond growth models might be able to reproduce observed (100) growth rates, but not (110) growth rates, (111) growth rates, or (100) surface morphologies. This hypothesis is supported by experimental evidence $\mathrm{e}^{27-30}$ that the majority of deposited $\mathrm{C}$ in $\mathrm{CVD}$ diamond comes from $\mathrm{CH}_{3}$. However, growth simulations ${ }^{25,26}$ demonstrate that $\mathrm{C}_{2} \mathrm{H}_{2}$ can be critical in promoting nucleation on the (110) and (111) surfaces, while representing only a minor fraction of the total deposited material. In terms of understanding the growth mechanisms, the (110) and (111) growth processes appear to be relatively straightforward, ${ }^{25,26,31,32}$ and it is the complex (100) growth process that has historically presented the most difficulty. ${ }^{19,22,33}$ Therefore, the second scenario is that diamond growth relies on both $\mathrm{CH}_{3}$ and possible small amounts of $\mathrm{C}_{2} \mathrm{H}_{2}$, and that current growth models contain a meaningful picture of (110) and (111) growth, but fail to adequately reproduce (100) growth behavior.

In this paper, we will reevaluate the mechanism for growth on the (100) surface of diamond to explain the slow growth of smooth (100) surfaces. To construct a generalized set of chemical surface reactions, we include the important chemistry from previous theoretical studies. ${ }^{19,34,35}$ In addition, we combine density functional theory (DFT) and semiempirical tight binding (TE) calculations to critically examine the chemistry of undercoordinated hydrocarbon complexes on the (100) surface of diamond. These calculations suggest that there exists a viable reaction pathway for the removal of isolated monomolecular methylene $\left(\mathrm{CH}_{2}\right)$ moieties from the (100) surface of diamond. Furthermore, the same calculations indicate that the removal of $\mathrm{CH}_{2}$ clusters from terrace and step edge sites is not appreciable. This "preferential etching" of isolated $\mathrm{CH}_{2}$ moieties from the (100) surface is assisted by atomic hydrogen, and effectively creates a barrier to (100) layer nucleation, which should slow the (100) growth rate. This allows relatively rapid $\mathrm{C}$ incorporation at step edges, which should produce smooth (100) surface features. Related mechanisms have been proposed in other studies, ${ }^{19,33,35,36}$ but either the etching kinetics were too slow to significantly affect growth, ${ }^{19}$ or the effects of the etching mechanism on diamond growth behavior were not explicitly determined. ${ }^{33,35,36}$ Our recent quantum mechanical calculations, on the other hand, indicate that a rapid etching mechanism does indeed exist. The precise effects of etching on the growth of the (100), (110), and (111) surfaces are ascertained using an atomic-scale kinetic Monte Carlo simulation technique. The growth rates of all three surface orientations and the morphology of the (100) surface are examined, and the agreement between the simulation predictions and experimental observations is discussed.

\section{DIAMOND GROWTH MODEL}

Diamond films are grown by the chemical deposition of hydrocarbon species in a vapor composed predominantly of hydrogen. Typical growth atmospheres in hot filament and microwave plasma-assisted CVD reactors contain mostly $\mathrm{H}_{2}$, with appreciable amounts of $\mathrm{H}, \mathrm{CH}_{3}, \mathrm{CH}_{4}$, and $\mathrm{C}_{2} \mathrm{H}_{2} .{ }^{7}$ Most of the unsatisfied $\mathrm{C}$ bonds on the growth surface are passivated by $\mathrm{H}$ atoms. Surface sites can be activated by the desorption or abstraction of $\mathrm{H}$ atoms, and growth at activated sites occurs by the chemisorption and subsequent incorporation of $\mathrm{CH}_{3}$ and $\mathrm{C}_{2} \mathrm{H}_{2}$. These and other chemical reactions are responsible for surface evolution and film growth. Since the chemical processes that are important to diamond CVD involve mainly $\mathrm{C}$ and $\mathrm{H}$, whose chemistry is well known from combustion studies, ${ }^{37}$ the rates can be easily estimated. $^{6,19}$ Though many surface reactions are possible, information about the concentrations of gas-phase species near the diamond surface and about the rates of the possible reactions allows the intractably large set of conceivable reactions to be reduced to a relatively small set of important ones.

This approach has been used in previous attempts ${ }^{19,20,22,23}$ to model the growth of diamond films. By making some basic assumptions about the process(es) by which diamond formation occurs from these species, the kinetics of diamond growth can be modeled. ${ }^{6,19,20}$ However, modeling of diamond growth using only the kinetics of surface reactions provides no explicit representation of the atomic-level processes by which chemisorbed hydrocarbons bond together and are converted to diamond. This limitation is particularly severe when the diamond formation process is complex, and overcoming it requires a realistic representation of the three-dimensional diamond cubic lattice in addition to the chemical kinetic information. Three-dimensional atomic-scale kinetic Monte Carlo simulations of diamond growth have already been applied to study the growth of specific diamond structures ${ }^{18}$ and particular surface orientations, ${ }^{22-24,26,38,39}$ and a comparison of the growth behavior of all three high-symmetry diamond surface orientations [(100), (110), and (111)] has been presented recently. ${ }^{40}$ The latter study clearly shows that the discrepancy between simulations and experimental observations of (100) growth remains largely unresolved.

In the present study, chemical kinetic information is combined with a three-dimensional atomic-scale representation of film growth to produce a diamond growth model that can handle arbitrary surface orientations and a wide range of surface chemical processes. The rate constants of the surface reactions and the concentrations of the gas-phase species are 
TABLE I. Chemical reactions, rate coefficients, and thermochemistry (Refs. 19, 20, and 35).

\begin{tabular}{|c|c|c|c|c|c|c|}
\hline & Reaction & $A$ & $n$ & E & $\Delta H$ & $\Delta S$ \\
\hline 1. & $\mathrm{C}_{d} \mathrm{H}+\mathrm{H} \leftrightarrow \mathrm{C}_{d}+\mathrm{H}_{2}$ & $1.3 \times 10^{14}$ & 0 & 7.3 & -9.9 & 5.3 \\
\hline 2. & $\mathrm{C}_{d}+\mathrm{H} \leftrightarrow \mathrm{C}_{d} \mathrm{H}$ & $1.0 \times 10^{13}$ & 0 & 0.0 & -96.9 & -32.8 \\
\hline 3. & $\mathrm{C}_{d} \mathrm{CH}_{2}+\mathrm{H} \leftrightarrow \mathrm{C}_{d}+\mathrm{CH}_{3}$ & $3.0 \times 10^{13}$ & 0 & 0.0 & -24.6 & 7.9 \\
\hline 4. & $\mathrm{C}_{d}+\mathrm{CH}_{3} \leftrightarrow \mathrm{C}_{d} \mathrm{CH}_{3}$ & $5.0 \times 10^{12}$ & 0 & 0.0 & -70.9 & -42.0 \\
\hline 5. & $\mathrm{C}_{d}+\mathrm{C}_{2} \mathrm{H}_{2} \leftrightarrow \mathrm{C}_{d} \mathrm{C}_{2} \mathrm{H}_{2}$ & $4.5 \times 10^{11}$ & 0 & 6.9 & -28.5 & -1.9 \\
\hline 6. & $\mathrm{C}_{d} \mathrm{CH}_{y}+\mathrm{H} \leftrightarrow \mathrm{C}_{d} \mathrm{CH}_{y-1}+\mathrm{H}_{2}$ & $2.8 \times 10^{7}$ & 2 & 7.7 & -11.3 & 6.6 \\
\hline 7. & $\mathrm{C}_{d} \mathrm{CH}_{y}+\mathrm{H} \leftrightarrow \mathrm{C}_{d} \mathrm{CH}_{y+1}$ & $1.0 \times 10^{13}$ & 0 & 0.0 & -83.0 & -34.1 \\
\hline 8. & $\mathrm{C}_{d} \mathrm{C}_{2} \mathrm{H}_{y}+\mathrm{H} \leftrightarrow \mathrm{C}_{d} \mathrm{C}_{2} \mathrm{H}_{y-1}+\mathrm{H}_{2}$ & $9.0 \times 10^{6}$ & 2 & 5.0 & -8.9 & 8.7 \\
\hline 9. & $\mathrm{C}_{d} \mathrm{C}_{2} \mathrm{H}_{y}+\mathrm{H} \leftrightarrow \mathrm{C}_{d} \mathrm{C}_{2} \mathrm{H}_{y+1}$ & $2.0 \times 10^{13}$ & 0 & 0.0 & -47.7 & -36.2 \\
\hline 10. & $\mathrm{C}_{d} \mathrm{C}_{2} \mathrm{H}_{y}+\mathrm{H} \leftrightarrow \mathrm{C}_{d} \mathrm{CH}_{y-2}+\mathrm{CH}_{3}$ & $3.0 \times 10^{13}$ & 0 & 0.0 & -24.6 & 7.9 \\
\hline 11. & $\mathrm{C}_{d} \mathrm{CH}_{y}+\mathrm{CH}_{3} \leftrightarrow \mathrm{C}_{d} \mathrm{C}_{2} \mathrm{H}_{y+3}$ & $5.0 \times 10^{12}$ & 0 & 0.0 & -70.9 & -42.0 \\
\hline 12. & $\mathrm{C}_{d} \cdots * \cdots \mathrm{C}_{d} \mathrm{C}_{x} \mathrm{H}_{y} \rightarrow \mathrm{C}_{d} \cdots \mathrm{C}_{d} \mathrm{C}_{x-1} \mathrm{H}_{y} \cdots \mathrm{C}_{d}$ & $2.0 \times 10^{13}$ & 0 & 8.8 & $\ldots$ & $\ldots$ \\
\hline 13. & $\mathrm{C}_{d} \cdot \mathrm{C}_{d} \mathrm{H}+\mathrm{H} \leftrightarrow \mathrm{C}_{d} \cdot \mathrm{C}_{d}+\mathrm{H}_{2}$ & $2.5 \times 10^{14}$ & 0 & 7.3 & -6.2 & 6.7 \\
\hline 14. & $\mathrm{C}_{d} \cdot \mathrm{C}_{d}+\mathrm{H} \leftrightarrow \mathrm{C}_{d} \cdot \mathrm{C}_{d} \mathrm{H}$ & $1.0 \times 10^{13}$ & 0 & 0.0 & -100.6 & -34.2 \\
\hline 15. & $\mathrm{C}_{d} \cdot \mathrm{C}_{d} \mathrm{CH}_{2}+\mathrm{H} \leftrightarrow \mathrm{C}_{d} \cdot \mathrm{C}_{d}+\mathrm{CH}_{3}$ & $3.0 \times 10^{13}$ & 0 & 0.0 & -17.8 & 8.0 \\
\hline 16. & $\mathrm{C}_{d} \cdot \mathrm{C}_{d}+\mathrm{CH}_{3} \leftrightarrow \mathrm{C}_{d} \cdot \mathrm{C}_{d} \mathrm{CH}_{3}$ & $5.0 \times 10^{12}$ & 0 & 0.0 & -81.0 & -42.2 \\
\hline 17. & $\mathrm{C}_{d} \cdot \mathrm{C}_{d} \leftrightarrow \mathrm{C}_{d} \cdots * \cdots \mathrm{C}_{d}$ & $1.0 \times 10^{13}$ & 0 & 0.0 & 4.9 & 0.4 \\
\hline 18. & $\mathrm{C}_{d} \cdots \mathrm{C}_{d} \mathrm{H}_{2} \cdots \mathrm{C}_{d}+\mathrm{H} \leftrightarrow \mathrm{C}_{d} \cdots * \cdots \mathrm{C}_{d}+\mathrm{CH}_{3}$ & $7.0 \times 10^{14}$ & 0 & 7.0 & $\ldots$ & $\ldots$ \\
\hline
\end{tabular}

$A$ is in moles, $\mathrm{cm}^{3}$, and s, as appropriate; $E$ and $\Delta H$ are in $\mathrm{kcal} \cdot \mathrm{mole}^{-1}$; and $\Delta S$ is in cal $\cdot \mathrm{mole}^{-1} \cdot \mathrm{K}^{-1}$.

used as input to a variable time step kinetic Monte Carlo algorithm, ${ }^{41,42}$ which simulates the evolution of the diamond growth surface by tracking the occupancies of surface sites. Dimer bonding, and the associated $\mathrm{C}$ incorporation mechanisms, e.g., $\beta$-scission and dimer/trough insertion, ${ }^{19,22,43}$ are included (Reactions 12 and 17 in Table I-see below). In the following discussion we focus mainly on the chemical reactions and the preferential etching mechanism used in this study, and the reader is referred to Ref. 38 for a detailed description of the kinetic Monte Carlo simulation method.

In order to describe the evolution and incorporation of surface species, we have adopted a set of surface chemical reactions and rate coefficients from several sources. ${ }^{19,34,35}$ These data are provided in Table I and involve interactions between $\mathrm{H}, \mathrm{H}_{2}, \mathrm{CH}_{3}, \mathrm{C}_{2} \mathrm{H}_{2}$, and the diamond surface. The information in Table I specifies the rates at which various species evolve on the growth surface. The calculation of forward and reverse rate constants from the data in Table I is accomplished in the standard fashion and is discussed elsewhere. ${ }^{38} C_{d}$ represents a surface diamond atom, species separated by a bullet $(\bullet)$ are dimer bonded, an asterisk $(*)$ represents a surface biradical, and an ellipsis (..) denotes species that are adjacent to one another on the diamond surface. Reactions 1 and 2 are between $\mathrm{H}$ and the diamond surface. Reactions 3-5 occur between hydrocarbon molecules and the surface. Reactions 6-9 are between $\mathrm{H}$ and chemisorbed hydrocarbons. Reactions 10 and 11 represent the addition of $\mathrm{CH}_{3}$ to an adsorbed hydrocarbon. Reactions 13-16 handle hydrogen and hydrocarbon molecules interacting with dimer-bonded surface atoms. (Reactions 5-11 apply to any surface site.) Reaction 17 represents formation/ breaking of dimer bonds between surface atoms, ${ }^{43}$ and Reaction 12 represents the insertion of a hydrocarbon into an opened dimer bond. ${ }^{43}$ Reaction 18 describes the etching of $\mathrm{C}$ from the surface of the film, as described in the following section. The reactions involving $\mathrm{H}$ and $\mathrm{CH}_{3}$ attachment to the surface (Reactions 1-11 and 13-16), and those describing dimer bonding (Reactions 12 and 17), were adopted from the work of Harris and Goodwin. ${ }^{19}$ The $\mathrm{C}_{2} \mathrm{H}_{2}$ kinetics ( $\mathrm{Re}$ action 5) were taken from the quantum mechanical calculations of Skokov et al. ${ }^{35}$

The reaction mechanism in Table I does not include the surface migration processes that have been proposed recently by Frenklach et al. ${ }^{35,44}$ The development of smooth (100) surface features could certainly be accomplished by supplementing the growth model with a mechanism for the diffusion and attachment of growth species to step edges. However, this would only serve to increase the predicted (100) growth rates (which are, as discussed above, already too high). Thus, while surface migration might be important on the (100) surface of diamond, ${ }^{44}$ it cannot provide an answer to the fundamental problems discussed above, and thus we have not considered it in the present study.

\section{SURFACE ETCHING MECHANISM}

In this study, we reexamined the details of the growth mechanisms specific to the (100) surface of diamond. The reaction pathway by which growth proceeds from $\mathrm{CH}_{3}$ additions on the $(100)(2 \times 1): \mathrm{H}$ surface is shown in Fig. 1(a). The reaction number (from Table I) is shown between each frame. Reactions 17 and 12 in Table I and Fig. 1(a) correspond to the $\beta$-scission and dimer insertion mechanisms proposed by Garrison et al. ${ }^{43}$ The combined two-step process allows C incorporation at the (100) surface, as shown in Fig. 1(b). The rate limiting step in the process is Reaction 12, since it is a factor of 20 slower than Reaction 17 at $1200 \mathrm{~K}$ (see Table I). The reverse of the incorporation process, followed by the removal of the adsorbed $\mathrm{CH}_{2}$ species via abstraction by atomic hydrogen, constitutes the "etching"' of undercoordinated $\mathrm{C}$ from the (100) surface, as shown in Fig. 1(c). In this study, we consider the etching reaction to be the composite process in Fig. 1(c). This is because the forward and reverse rates of Reactions 12 and 17 are very fast compared to the other reactions on the surface, and explicitly 


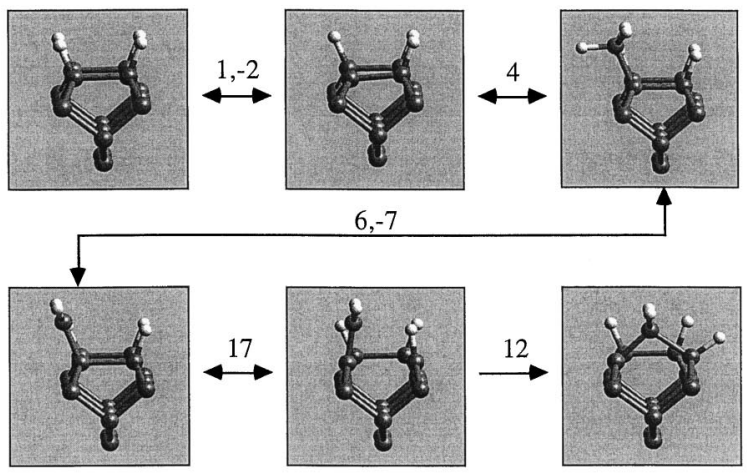

(a)

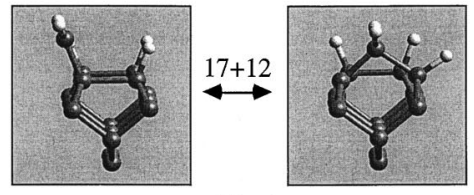

(b)

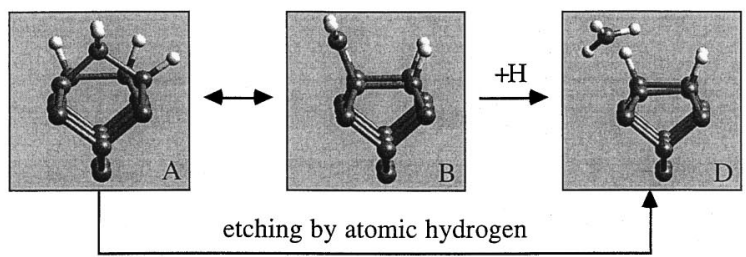

(c)

FIG. 1. Reactions involving (a) growth, (b) C incorporation, and (c) etching on the $(100)(2 \times 1)$ surface. Gray atoms are carbons and white atoms are hydrogens.

incorporating each step from Fig. 1(c) into the kinetic Monte Carlo growth simulation would be cumbersome.

Harris and Goodwin ${ }^{19}$ estimated a Gibbs free energy change, $\Delta G_{12}$, for dimer insertion (Reaction 12) of approximately $\Delta G_{12}=-21.2 \mathrm{kcal} \cdot \mathrm{mole}^{-1}$ at $1200 \mathrm{~K}$, indicating that the forward reaction dominates the reverse. This was the justification for assuming the insertion reaction to be irreversible in previous studies. ${ }^{38}$ The thermochemistry adopted by Harris and Goodwin was obtained by molecular mechanics (MM3) calculations, which are prone to significant errors when applied to hydrocarbon radicals. ${ }^{19}$ If the thermochemistry associated with $\mathrm{C}$ incorporation was such that the rate of the reverse of incorporation (and, therefore, etching) were appreciable, then growth on the (100) surface could be significantly affected. Therefore, we have reevaluated the energetics associated with $\mathrm{C}$ incorporation at the (100) surface using more accurate quantum mechanical methods, as described below.

\section{A. Etching kinetics}

The reaction sequence depicted in Fig. 1(c) can be expressed as

$$
\begin{aligned}
& \underset{k_{-\mathrm{I}}}{\mathrm{A} \rightleftarrows \mathrm{B},} \\
& \mathrm{B}+\mathrm{H} \rightarrow \mathrm{D} \rightarrow \mathrm{CH}_{3},
\end{aligned}
$$

where Reaction I denotes the reverse of the combined $\beta$-scission and dimer insertion processes, i.e., the reverse of Reactions 17 and 12, as discussed above. Reaction II is identical to Reaction 3 in Table I, and is assumed irreversible in Eq. (II) due to the large negative Gibbs free energy change, approximately $\Delta G_{\mathrm{II}}=-30 \mathrm{kcal} \cdot \mathrm{mole}{ }^{-1},{ }^{19}$ associated with the reaction. Our quantum mechanical calculations (see below) indicate that Reaction I proceeds at similar rates in the forward and reverse directions, and thus we can assume steady state for the reaction subsystem in Eqs. (I) and (II). Thus, the system of kinetic equations associated with the reaction subsystem reduces to

$$
\begin{aligned}
& k_{\mathrm{I}}[\mathrm{A}]=k_{-\mathrm{I}}[\mathrm{B}], \\
& \frac{d[\mathrm{D}]}{d t}=k_{\mathrm{II}}[\mathrm{B}][\mathrm{H}],
\end{aligned}
$$

where $k_{\mathrm{I}}, k_{-\mathrm{I}}$, and $k_{\mathrm{II}}$ are the relevant reaction rate constants for Eqs. (I) and (II); [A], [B], and [D] are the concentrations of the species depicted in Fig. 1(c); and $[\mathrm{H}]$ is the concentration of atomic hydrogen. Thus, the per-site rate of etching, $r_{e}$, is

$$
r_{e}=\frac{1}{[\mathrm{~A}]} \frac{d[\mathrm{D}]}{d t}=K_{\mathrm{I}}^{\mathrm{eq}} k_{\mathrm{II}}[\mathrm{H}],
$$

where $K_{\mathrm{I}}^{\mathrm{eq}}$ is the equilibrium constant for Reaction I, i.e.,

$K_{\mathrm{I}}^{\mathrm{eq}} \equiv \frac{k_{\mathrm{I}}}{k_{-\mathrm{I}}}=\exp \left(-\frac{\Delta G_{\mathrm{I}}}{R T}\right)=\exp \left(\frac{\Delta S_{\mathrm{I}}}{R}\right) \exp \left(-\frac{\Delta H_{\mathrm{I}}}{R T}\right)$,

where $\Delta G_{\mathrm{I}}, \Delta H_{\mathrm{I}}$, and $\Delta S_{\mathrm{I}}$ are the Gibbs free energy, enthalpy, and entropy changes, respectively, for Reaction I. Substituting Eq. (4) into Eq. (3) yields

$$
r_{e}=k_{\mathrm{II}}[\mathrm{H}] \exp \left(\frac{\Delta S_{\mathrm{I}}}{R}\right) \exp \left(-\frac{\Delta H_{\mathrm{I}}}{R T}\right) .
$$

The rate constant, $k_{e}$, for the etching reaction is obtained by dividing the absolute rate, $r_{e}$, by the concentration of the appropriate gas-phase reactant, ${ }^{38}$ i.e., $[\mathrm{H}]$, which yields

$$
k_{e}=k_{\mathrm{II}} \exp \left(\frac{\Delta S_{\mathrm{I}}}{R}\right) \exp \left(-\frac{\Delta H_{\mathrm{I}}}{R T}\right) .
$$

Since the rate of Reaction II is approximately independent of temperature (see Reaction 3 in Table I), and $\Delta S_{\mathrm{I}}$ is small and only weakly dependent on temperature, ${ }^{45}$ the temperature dependence of the etching reaction rate in Eq. (6) is defined by the enthalpy change of the incorporation Reaction I. Therefore, the heat of Reaction I, $\Delta H_{\mathrm{I}}$, serves as an effective activation barrier for the net etching reaction, and it represents a parameter that is critical to (100) growth behavior and must be quantified as precisely as possible.

\section{B. Etching thermochemistry}

In order to estimate the thermochemistry associated with Reaction I, we performed quantum mechanical calculations of the energetics of the relevant surface configurations. Since the forming and breaking of bonds at the surface is accompanied by substantial rearrangements of the atoms around the reacting molecules, we have employed a scheme wherein the 
TABLE II. Thermochemistry of Reaction I [see Eq. (I)] at $1200 \mathrm{~K}$, as calculated by TB/DFT, MM3 (Ref. 19), and PM3 (Ref. 55) approaches. $\Delta S$ is in $\mathrm{cal} \cdot \mathrm{mole}^{-1} \cdot \mathrm{K}^{-1}$, and $\Delta G$ and $\Delta H$ are in $\mathrm{kcal} \cdot \mathrm{mole}^{-1}$.

\begin{tabular}{lcrc}
\hline \hline Method & $\Delta S_{1}$ & $\Delta H_{1}$ & $\Delta G_{1}$ \\
\hline TB/DFT & 6.4 & 7.0 & -0.7 \\
MM3 & 5.4 & 23.4 & 16.9 \\
PM3 & 0.7 & 11.6 & 10.8 \\
\hline \hline
\end{tabular}

region of interest is divided into two parts. A small volume of approximately 20 carbon atoms located at the reaction zone is treated by density functional theory (DFT). A larger volume that includes the reaction zone itself is modeled by the semiempirical tight binding (TB) method. The TB slab consists of five atomic layers with 16 atoms in each layer. The forces on the atoms in the total system are composed of the DFT forces acting on the atoms in the reaction zone plus the TB forces acting on the atoms in the diamond slab. The TB forces in the reaction region are replaced by corresponding DFT forces. The atomic positions of all the atoms in the combined system are relaxed simultaneously to have forces acting on each atom equal to zero. The DFT calculations were preformed using the MSI density functional code, DMOL ${ }^{46}$ with Becke- $1988^{47}$ gradient-corrected exchange and Perdew-Wang ${ }^{48}$ gradient-corrected correlation functionals. The TB region was handled by a two-center orthogonal tight binding parametrization ${ }^{49-51}$ for hydrocarbons, as implemented in the oxON code. ${ }^{52}$ Our approach of combining quantum mechanical calculations with less accurate semiempirical methods is similar to the scheme pioneered by Frenklach et al. ${ }^{53}$

The interface between the TB and DFT regions of the slab is handled in the following manner. The forces on the atoms in the DFT region are calculated by "cutting" the cluster from the larger diamond slab and saturating the broken $\mathrm{C}-\mathrm{C}$ bonds with hydrogen atoms. The positions of these hydrogens are chosen to take into account the proper changes in the nearest-neighbor environment around each carbon atom at the TB/DFT boundary during the structural relaxation. As the total system is relaxed, the cluster shape evolves to match the displacements of the carbon atoms in the TB slab. We have checked that the TB and DFT forces are consistent at the TB/DFT boundary, and that the size of the cluster is sufficient to provide an accurate description of the local surface chemistry. The details of the combined TB/ DFT approach can be found in Ref. 54, and a more complete description of its application to the study of diamond (100) surface chemistry will be reported in a future publication.

The thermochemistry obtained for Reaction I by the combined TB/DFT approach is provided in Table II. Estimates from molecular mechanics (MM3) ${ }^{19}$ and parametrized model $(\mathrm{PM} 3)^{44,56,55}$ calculations are provided for comparison. The TB/DFT method predicts a heat of reaction, $\Delta H_{\mathrm{I}}$, that is three times smaller than that estimated by Harris and Goodwin ${ }^{19}$ and an entropy change, $\Delta S_{\mathrm{I}}$, that is nearly the same. The TB/DFT value for $\Delta H_{\mathrm{I}}$ agrees well with the PM3 calculations of Frenklach et al. ${ }^{44,56,55}$ We estimated the PM3 value for $\Delta S_{\mathrm{I}}$ using the kinetic simulations of Frenklach et al., ${ }^{44,56,55}$ who did not report this quantity. $\mathrm{A} \mathrm{CH}_{2}$ adsorbate is expected to lose some rotational and translational degrees of freedom when it is incorporated at the surface [see Fig. 1(b)] and thus an appreciable positive entropy change is expected for Reaction I, so it is not clear why the PM3 approach should yield a negligible value for $\Delta S_{\mathrm{I}}$.

The most important result from the data in Table II is that Reaction I involves a very small change in Gibbs free energy, and can thus proceed at similar rates in both directions. The fact that the reverse of Reaction I is very fast [see Fig. 1(b), and Reactions 12 and 17 in Table I] implies that there exists a viable reaction path for the removal of inserted $\mathrm{C}_{d} \mathrm{H}_{2}$ molecules by Reactions I and II, as in Fig. 1(c). (The notation, $\mathrm{C}_{d}$, distinguishes inserted $\mathrm{C}$ complexes from adsorbed methylene, which is labeled $\mathrm{CH}_{2}$.) The rate constant (in units of moles $\cdot \mathrm{cm}^{-3} \cdot \mathrm{s}^{-1}$ ) for the combined etching reaction shown in Fig. 1(c) can be obtained by substituting the TB/DFT values from Table II and the rate constant for Reaction 3 from Table I into Eq. (6), which yields

$$
k_{e}=7 \times 10^{14} \exp \left(\frac{-7}{R T}\right),
$$

where $R$ is the universal gas constant, i.e., 1.987 $\mathrm{kcal} \cdot \mathrm{mole}^{-1} \cdot \mathrm{K}^{-1}$, and $T$ is in units of $\mathrm{K}$. The kinetics for Reaction 3 in Table I were estimated by Harris and Goodwin ${ }^{19}$ from analogous gas-phase chemistry, ${ }^{57}$ and are believed to be sufficiently accurate for the present purpose. The absolute rate (in units of $\mathrm{s}^{-1}$ ) of the etching reaction is obtained by multiplying Eq. (7) by the gas-phase concentration of atomic hydrogen, ${ }^{38}$ i.e., $[\mathrm{H}]$, as described above.

\section{Etching configurations}

The results presented in Table II and Eq. (7) indicate that the removal of $\mathrm{C}_{d} \mathrm{H}_{2}$ from the (100) surface of diamond occurs at an appreciable rate. The inclusion of a kinetically viable etching mechanism to the diamond growth model is practically guaranteed to reduce the growth rate. However, the magnitude of this effect is difficult to gauge a priori, and the impact on the growth of the other faces [i.e., (110) and (111)] must be ascertained. In addition, etching by itself does nothing to promote the growth of smooth surfaces. Just as C incorporation can occur anywhere on the (100) surface with equal probability, ${ }^{25,26}$ so can etching. Thus the simplest etching mechanism would slow the growth rate, but could not generate smooth surfaces. In order to reconcile the growth model with experimental observations, growth must occur preferentially at step edges to promote smooth surface features. We have used the TB/DFT method described above to investigate the energetics of etching from different surface structures. These structures are depicted in Fig. 2, and correspond to an isolated monomolecular $\mathrm{C}_{d} \mathrm{H}_{2}$ [Fig. 2(a)], a $\mathrm{C}_{d} \mathrm{H}_{2}$ on a type $S_{B}$ step edge ${ }^{58}$ [Fig. 2(b)], a $\mathrm{C}_{d} \mathrm{H}_{2}$ in the midst of a flat (100) terrace [Fig. 2(c)], and a dimer bonded $\mathrm{CH}$ [Fig. 2(d)]. According to our calculations, the enthalpy change upon etching (by Reactions I and II) is about $\Delta H_{e}$ $=-7.8 \mathrm{kcal} \cdot \mathrm{mole} \mathrm{e}^{-1}$ when the etched $\mathrm{C}$ atom is alone on the surface [Fig. 2(a)], $\Delta H_{e}=9.4 \mathrm{kcal} \cdot \mathrm{mole}^{-1}$ when the etched atom is at the edge of a type $S_{B}$ step [Fig. 2(b)], and $\Delta H_{e}$ 


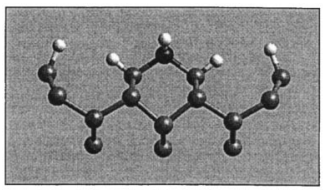

(a)

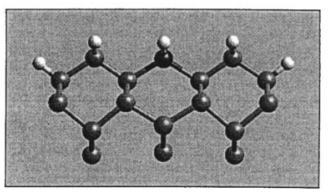

(c)

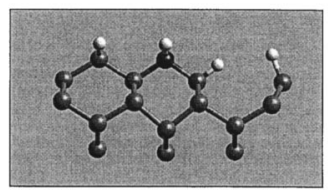

(b)

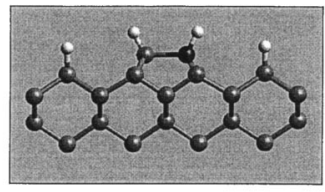

(d)
FIG. 2. Examples of atomic structures that (a) can and (b), (c), (d) cannot be etched from the $(100)(2 \times 1)$ surface of diamond. Light gray atoms are carbons, dark gray atoms are the carbons to be considered for etching, and white atoms are hydrogens.

$=57.7 \mathrm{kcal} \cdot \mathrm{mole}^{-1}$ when the atom is in the midst of a surface terrace [Fig. 2(c)]. When the dark atom in Fig. 2(a) is etched, the biradical that is left behind is free to relax and reduce its energy. However, when the surrounding diamond lattice is constrained by other inserted $\mathrm{C}_{d} \mathrm{H}_{2}$ molecules, as in Figs. 2(b) and 2(c), the biradical at the etched site cannot relax, and is therefore high in energy. Thus the energy required to remove a $\mathrm{C}_{d} \mathrm{H}_{2}$ complex from the (100) surface is much higher when the complex is adjacent to other $\mathrm{C}_{d} \mathrm{H}_{2}$ units. This suggests that etching is much more likely to occur at an isolated $\mathrm{C}_{d} \mathrm{H}_{2}$ than at a step edge or on a flat face. Therefore, etching in our simulations is only allowed to occur at $\mathrm{C}_{d} \mathrm{H}_{2}$ complexes that are isolated monomolecular islands on the (100) surface, as in Fig. 1(a). $\mathrm{C}_{d} \mathrm{H}_{2}$ molecules that are part of a larger island like those in Figs. 1(b) and 1(c) are not allowed to etch. Furthermore, the removal of a dimer bonded $\mathrm{C}$ atom involves the breaking of three $\mathrm{C}-\mathrm{C}$ bonds, which should involve a very large increase in energy, and thus dimer bonded $\mathrm{C}$ atoms [Fig. 1(d)] are also prohibited from etching. In terms of the simulation methodology, these etching criteria dictate that a diamond atom can only be etched if (1) it is not dimer bonded, (2) it is bonded to exactly two other diamond atoms, and (3) these two diamond neighbors have exactly three diamond neighbors each. These requirements describe the etching of a lone $\mathrm{C}_{d} \mathrm{H}_{2}$ moiety on the (100) surface, and ensure that atoms that are part of a larger island will not be etched, as indicated by our TB/DFT calculations.

It should be noted that this etching scheme contains some similarities to the growth model of Harris and Goodwin. ${ }^{19}$ In their reaction mechanism, diamond growth starts by dimer insertion (as in Fig. 1), and continues by insertion into an adjacent "trough" (i.e., a site between two dimers). They assumed that dimer insertion is reversible in principle (though very slow in the reverse direction in practice), and that insertion into a "trough" (i.e., a site between two dimers) is not. Since their growth mechanism demands that dimer insertion occur first, the reversibility of the dimer insertion reaction implies that isolated $\mathrm{C}_{d} \mathrm{H}_{2}$ can be etched from the surface, just as we have assumed here [see Fig. 2(c)]. In addition, since their mechanism requires that trough insertion occur only after, and adjacent to, a dimer insertion event, the irreversibility of trough insertion implies that type $S_{B}$ step edge sites (and, by extension, terrace sites) cannot be etched. This, of course, is the same assumption made here [see Fig. 2(a)]. [Our Figs. 2(a), 2(b), and 2(d) can be compared to Harris' and Goodwin's Figs. 3(c), 3(f), and 3(a), respectively.] However, despite these similarities in the two molecular pictures of etching, the reaction kinetics and thermochemistry that Harris and Goodwin ascribed to their dimer insertion reaction (see above) were not sufficient to dramatically alter the growth behavior. ${ }^{59}$

\section{RESULTS}

To gauge the effects of anisotropic etching on diamond growth, kinetic Monte Carlo simulations of growth on the (100), (110), and (111) surfaces were performed at substrate temperatures between 800 and $1400 \mathrm{~K}$. The gas near the growth surface was assumed to contain constant partial pressures of 0.05 Torr $\mathrm{H}, 18$ Torr $\mathrm{H}_{2}, 0.002$ Torr $\mathrm{CH}_{3}$, and 0.05 Torr $\mathrm{C}_{2} \mathrm{H}_{2}$, which is typical of hot filament CVD reactors. ${ }^{3,7}$ These pressures are assumed constant, and are used to obtain absolute reaction rates from the rate coefficients in Table I, as described in Ref. 38. The growth rates on the (100), (110), and (111) surfaces were calculated and compared with available experimental values, and the (100) surface morphologies were examined.

The growth rates of all three surface orientations as functions of temperature are shown in Fig. 3 for simulations with and without etching. Data from experiments are included for comparison. The $(+)$ symbols are single crystal growth rates from the measurements of Chu et al. ${ }^{16}(0.4 \%$ $\mathrm{CH}_{4}$ in $\mathrm{H}_{2}$ fed into a hot filament reactor held at 25 Torr), and the $(\times)$ symbols in Fig. 3(a) are (100) growth rates from Rawles et al. ${ }^{17}\left(0.5 \% \mathrm{CH}_{4}\right.$ in $\mathrm{H}_{2}$ fed into a hot filament reactor held at 25 Torr). When the growth simulations are performed without etching, the (100) growth rate [Fig. 3(a)] is highest, followed by (110) [Fig. 3(b)], and the (111) growth rate [Fig. 3(c)] is lowest. Obviously, this is in contradiction with experiments. ${ }^{16}$ Even without etching, the magnitudes of the (110) and (111) growth rates are in reasonable agreement with experimental values [Figs. 3(b) and 3(c)], but the (100) growth rates are not [Fig. 3(a)]. The (100) growth rate is reduced substantially by including etching in the growth model, as shown in Fig. 3(a). The (110) and (111) growth rates change only slightly due to etching because even rough surfaces in these orientations contain very few sites resembling an isolated $\mathrm{C}_{d} \mathrm{H}_{2}$ molecule on the (100) surface. [The subtle differences between the two sets of simulated (111) growth rates in Fig. 3(c) are due primarily to statistical variations in the stochastic Monte Carlo procedure.] Thus, when the etching mechanism is included in the growth model, the absolute and relative magnitudes of the growth rates on all three surfaces are brought into reasonable agreement with the experimental measurements.

An example of the rough atomic surface morphologies of simulated (100) films grown without etching is shown in Fig. 4(a). As discussed above, numerous experiments ${ }^{11-13}$ show that (100) facets on the CVD diamond are atomically smooth. The etching mechanism presented in this paper pro- 


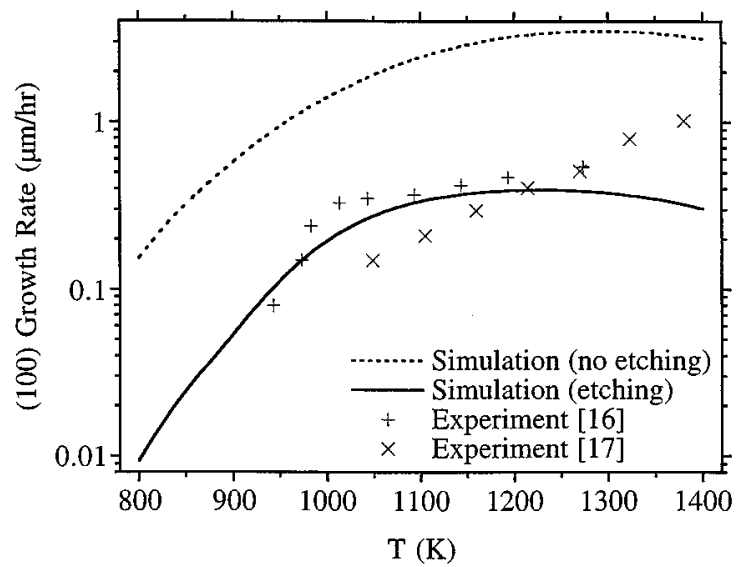

(a)

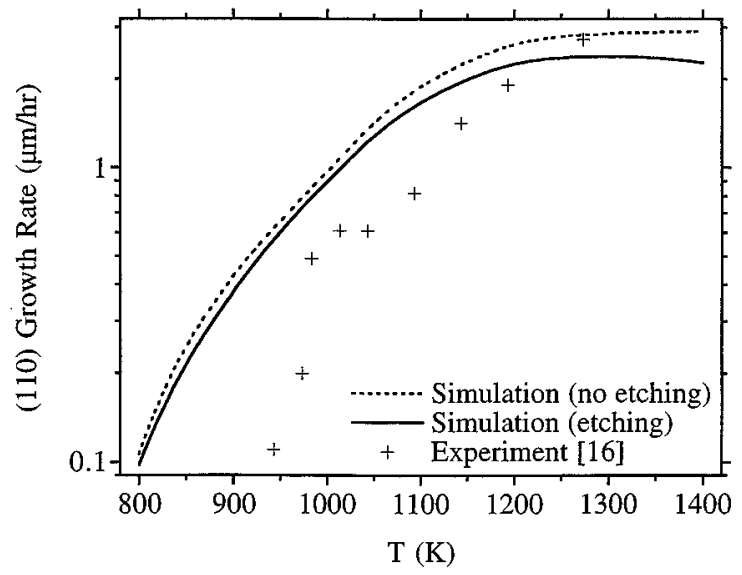

(b)

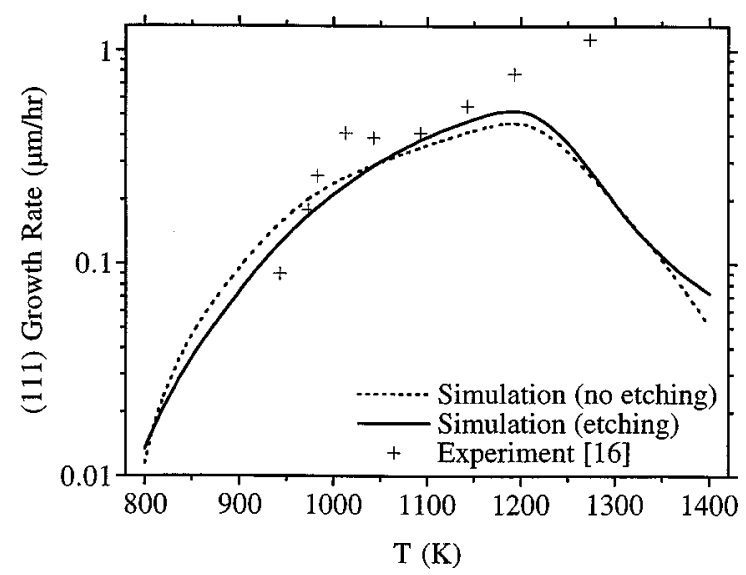

(c)

FIG. 3. Growth rates of (a) (100), (b) (110), and (c) (111) films as functions of substrate temperature. The dotted lines are simulations values with no etching, the solid lines are simulations values with etching, and the $(+)$ and $(\times)$ symbols are experimental values provided for comparison.

motes the incorporation of $\mathrm{C}$ at step edges on the (100) surface to produce smooth surface features. This is demonstrated in Fig. 4(b), which contains an image of the atomic surface morphology produced when etching is active. The (100) surface in Fig. 4(b) shows the relatively smooth, terrace/step morphology commonly associated with step flow growth.

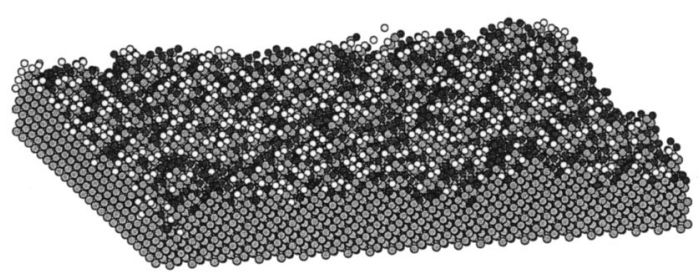

(a)

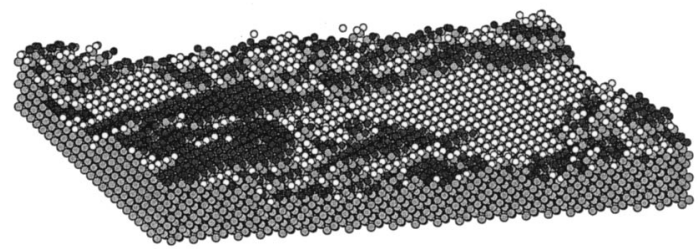

(b)

FIG. 4. Images of (100) films during growth at $1200 \mathrm{~K}$ (a) without and (b) with etching. Light gray atoms are carbons in the diamond film. The hydrogen atoms are shaded according to their height. Two gray levels (dark gray and white) are used, and cycle every two layers.

\section{DISCUSSION}

The results presented above suggest that much of the experimentally observed CVD diamond growth behavior on the (100) surface can be reproduced reasonably well if the etching of isolated $\mathrm{C}_{d} \mathrm{H}_{2}$ moieties from the (100) surface is considered. Nonetheless, there are some differences between the growth behavior observed experimentally and that predicted by the theory. The simulated growth rates exhibit maxima around $1200 \mathrm{~K}$, whereas the experimental measurements of single-crystal growth rates ${ }^{16,17}$ do not show such a pronounced peak. On the other hand, measurements of polycrystalline growth rates do show maxima with temperature ${ }^{60,61}$ but this might arise due to the deposition of nondiamond carbon at high temperatures in these experiments. ${ }^{60}$ In the simulations, the growth rates decrease at high temperatures because $\mathrm{CH}_{3}$ and $\mathrm{C}_{2} \mathrm{H}_{2}$ desorption becomes more rapid, and competes with the carbon incorporation reactions. The "turnover" in the (100) growth rate represented by the solid curve in Fig. 1(a) is exacerbated by etching at high temperatures. This discrepancy between the simulated and experimental temperature behavior in singlecrystal growth might be due to the influence of other phenomena such as twinning or specific defect-mediated surface chemistry that are present in the real experiments but absent from the growth model.

Despite this fact, the overall success of the current growth model in predicting the relative growth rates on (100), (110), and (111), and at reproducing some of the features of the (100) morphology, is encouraging. This work, and our earlier studies, ${ }^{25,26}$ suggest that $\mathrm{C}_{2} \mathrm{H}_{2}$ (or more generically, $\mathrm{C}_{2} \mathrm{H}_{x}$, where $x=0-6$ ) at the growth surface is an important growth species, particularly in that it assists the nucleation of next-layer growth. However, it is worth mentioning that the reliance of (110) and (111) growth on $\mathrm{C}_{2} \mathrm{H}_{2}$ does not imply that a significant fraction of $\mathrm{C}_{2} \mathrm{H}_{2}$ becomes incorporated into the film. In fact, our simulations ${ }^{25,26}$ suggest that $\mathrm{C}_{2} \mathrm{H}_{2}$ is necessary to promote layer nucleation on the (110) and (111) surfaces, but that only a few percent of 
the deposited $\mathrm{C}$ comes from $\mathrm{C}_{2} \mathrm{H}_{2}$, in agreement with experiments. ${ }^{27-29}$ Furthermore, the reliance of (110) and (111) growth on $\mathrm{C}_{2} \mathrm{H}_{2}$ applies only to single-crystal deposition. Although the mechanisms of growth on the individual surfaces are important building blocks in our knowledge of diamond CVD, the real CVD experiments are much more complicated. Diamond films are often polycrystalline and/or multifaceted. In this case, it is likely that features unique to the polycrystal, e.g., $\{100\} /\{111\}$ facet intersections, might significantly alter the nucleation mechanisms over the single crystal case, and could eliminate the need for layer nucleation on the (111) surface. Therefore, the presence of $\mathrm{C}_{2} \mathrm{H}_{2}$ might not be necessary for the growth of (111) facets on a polycrystal, and our computational growth studies cannot directly confirm the experimental suggestions ${ }^{62,63}$ that polycrystalline CVD diamond can be grown from $\mathrm{CH}_{3}$ alone.

Though the proposed etching mechanism is supported by reliable quantum mechanical calculations, we cannot dismiss the possibility that other channels exist for the removal of hydrocarbons from the surface in the CVD chemical environment. Also, there may be other surface processes that play an important role in the development of smooth $(100)(2 \times 1)$ surface features. For example, short-range diffusion and attachment of $\mathrm{CH}_{2}$ to step edges may occur, 35,44 or the chemical reaction kinetics $\left(\mathrm{H}\right.$ and $\mathrm{CH}_{3}$ addition and removal) on the (100) surface might promote growth at steps. Nonetheless, etching as a surface chemical process is thermodynamically sound and works very effectively to smooth (100) surfaces and to slow (100) growth rates while leaving (110) and (111) growth rates relatively unaffected, in agreement with experiments.

There are some important limitations of the growth model presented here that deserve mention. For example, the present model is incapable of predicting the well-ordered $(100)(2 \times 1)$ dimer row reconstructed domains that are observed experimentally. ${ }^{11-13}$ As stated above, observations of smooth surface features on (100) diamond indicate preferential growth at step edges, which can be accomplished by the etching mechanism used here. The presence of ordered $(100)(2 \times 1)$ surface material suggests that, when new material deposits at a step edge, it "prefers" to form dimer bonds in registry with the existing dimer row pattern. This might arise due to the strain imposed on the diamond lattice by the existing dimer rows, or from a stereochemical effect that promotes growth of dimer-bonded $\mathrm{C}$ pairs in registry with the existing reconstruction. Furthermore, it is possible that short-range diffusion of dimer bonds (via $\mathrm{CH}_{2}$ migration $)^{35}$ plays a role in facilitating the alignment of the dimer row pattern. Experimental evidence ${ }^{64}$ also suggests that much of the smooth, ordered $(100)(2 \times 1)$ material observed in the experiments might not be produced during growth, but rather by hydrogen-assisted surface diffusion or etching/regrowth in a $\mathrm{H} / \mathrm{H}_{2}$ plasma prior to scanning probe analysis. In addition, (100) faces on CVD diamond often show macrosteps, ${ }^{65,66}$ presumably from step bunching, which suggests that some mechanism (perhaps nitrogen assisted) exists to pin (100) steps as they grow. Thus the precise mechanism(s) that lead to the development of smooth, ordered $(100)(2 \times 1)$ domains, and their importance during the
CVD process itself, remain unclear. However, the results of the present study demonstrate that preferential etching of (100) surface features accounts for the experimental observations of slow-growing smooth (100) faces.

\section{CONCLUSIONS}

Chemically vapor deposited (100)-oriented diamond is known to grow more slowly than (110)-oriented material and to contain smooth surface features. The existing selfconsistent models for diamond growth, on the other hand, predict that (100)-oriented films grow fastest and are atomically rough. Surfaces that grow slowly and develop smooth features, such as (111), do so because the nucleation of new growth layers on these surfaces is much slower than growth at step edges, but previous growth models contain no nucleation barrier for the formation of new growth layers on (100) surfaces. This suggests that the experimentally observed (100) growth behavior might be rationalized by the introduction of growth mechanisms that hinder layer nucleation and promote step-flow growth. One such mechanism is the preferential etching of monomolecular carbon islands. The thermochemistry of the (100) dimer insertion reaction, by which $\mathrm{CH}_{2}$ species are incorporated at the surface, indicates that the incorporation reaction is reversible and that an etching mechanism does indeed exist.

We have presented the results of combined tight binding (TB) and density functional theory (DFT) quantum mechanical calculations that suggest that the etching of isolated, monomolecular moieties occurs at an appreciable rate, while etching from larger carbon islands (i.e., step edges and terraces) is not favorable. The TB/DFT calculations and the proposed etching mechanism were combined with previously determined hydrocarbon reaction rates on diamond surfaces and input to a kinetic Monte Carlo model of the chemical vapor deposition of diamond on (100), (110), and (111) surfaces. The simulation results clearly demonstrate that the inclusion of etching produces much smoother (100) diamond surfaces as compared with calculations that omit etching. Etching also slows (100) growth considerably without substantially affecting (110) and (111) growth rates. The growth rates that are predicted when etching is included in the model are in very good agreement with a wide range of experimental evidence that suggests that (100) growth is slower than (110) growth and comparable to (111) growth under hot filament CVD reactor conditions. However, while etching is key to our understanding of diamond growth in general, and (100) growth in particular, several details of (100) growth still need to be addressed in future studies.

\section{ACKNOWLEDGMENTS}

The authors wish to thank D. S. Dandy, M. P. D'Evelyn, and M. E. Frenklach for many enlightening discussions. The authors gratefully acknowledge the support of the US Defense Advanced Research Projects Agency (DARPA), the US Naval Research Laboratory (NRL), and the US Department of Energy (DOE). This work was performed at the University of Michigan under DARPA and NRL Contract Nos. N00014-97-1-G013 and N00014-96-1-G000; at Oxford 
University under DARPA and NRL Contract Nos. N0001497-1-G015 and N00014-96-G001; and at Sandia National Laboratories under DOE Contract No. DE-AC0494AL85000. The TB/DFT computations were performed on a Hewlett-Packard Exemplar parallel computer and on a SGI Cray Origin-2000 supercomputer at the Oxford Supercomputer Centre, jointly funded by Hewlett-Packard, Silicon Graphics UK Ltd., and the Higher Education Funding Council for England (HEFCE) Joint Research Equipment Initiative.

${ }^{1}$ D. G. Goodwin and J. E. Butler, in Handbook of Industrial Diamonds and Diamond Films, edited by M. A. Prelas, G. Popovici, and L. K. Bigelow (Dekker, New York, 1997) pp. 527-582.

${ }^{2}$ F. G. Celii, P. E. Pehrsson, H.-t. Wang, and J. E. Butler, Appl. Phys. Lett. 52, 2043-2045 (1988).

${ }^{3}$ W. L. Hsu, Appl. Phys. Lett. 59, 1427-1429 (1991).

${ }^{4}$ F. G. Celii and J. E. Butler, J. Appl. Phys. 71, 2877-2883 (1992).

${ }^{5}$ M. C. McMaster, W. L. Hsu, M. E. Coltrin, D. S. Dandy, and C. Fox, Diamond Relat. Mater. 4, 1000-1008 (1995).

${ }^{6}$ M. Frenklach and H. Wang, Phys. Rev. B 43, 1520-1545 (1991).

${ }^{7}$ D. S. Dandy and M. E. Coltrin, J. Appl. Phys. 76, 3102-3113 (1994).

${ }^{8}$ K. E. Spear, J. Am. Ceram. Soc. 72, 171-191 (1989).

${ }^{9}$ C. Wild, P. Koidl, W. Müller-Sebert, H. Walcher, R. Kohl, N. Herres, R. Locher, R. Samlenski, and R. Brenn, Diamond Relat. Mater. 2, 158-168 (1993).

${ }^{10}$ A. Badzian and T. Badzian, Diamond Relat. Mater. 2, 147-157 (1993).

${ }^{11}$ H. Sasaki, M. Aoki, and H. Kawarada, Diamond Relat. Mater. 2, 12711276 (1993).

${ }^{12}$ Y. Kuang, Y. Wang, N. Lee, A. Badzian, T. Badzian, and T. T. Tsong, Appl. Phys. Lett. 67, 3721-3723 (1995).

${ }^{13}$ B. D. Thoms and J. E. Butler, Surf. Sci. 328, 291-301 (1995).

${ }^{14}$ G. Janssen, J. J. Schermer, W. J. P. v. Enckevort, and L. J. Giling, J. Cryst. Growth 125, 42-50 (1992).

${ }^{15}$ K. A. Snail, Z. P. Lu, R. Weimer, J. Heberlein, E. Pfender, and L. M. Hanssen, J. Cryst. Growth 137, 676-679 (1994).

${ }^{16}$ C. J. Chu, R. H. Hauge, J. L. Margrave, and M. P. D’Evelyn, Appl. Phys. Lett. 61, 1393-1395 (1992).

${ }^{17}$ R. E. Rawles, W. G. Morris, and M. P. D'Evelyn, in Diamond for Electronic Applications, edited by D. L. Dreifus, A. Collins, T. Humphreys, K. Das, and P. E. Pehrsson, Mater. Res. Soc. Symp. Proc. 416 (MRS, Pittsburgh, 1996), pp. 13-18.

${ }^{18}$ M. Frenklach, J. Chem. Phys. 97, 5794-5802 (1992).

${ }^{19}$ S. J. Harris and D. G. Goodwin, J. Phys. Chem. 97, 23-28 (1993).

${ }^{20}$ M. E. Coltrin and D. S. Dandy, J. Appl. Phys. 74, 5803-5820 (1993).

${ }^{21}$ D. S. Dandy and M. E. Coltrin, J. Mater. Res. 10, 1993-2010 (1995).

${ }^{22}$ E. J. Dawnkaski, D. Srivastava, and B. J. Garrison, J. Chem. Phys. 104, 5997-6008 (1996).

${ }^{23}$ M. M. Clark, L. M. Raff, and H. L. Scott, Comput. Phys. 10, 584-590 (1996).

${ }^{24}$ C. C. Battaile, D. J. Srolovitz, and J. E. Butler, J. Electron. Mater. 26, 960-965 (1997).

${ }^{25}$ C. C. Battaile, D. J. Srolovitz, and J. E. Butler, J. Cryst. Growth 194, 353-368 (1998).

${ }^{26}$ C. C. Battaile, D. J. Srolovitz, and J. E. Butler, Diamond Relat. Mater. 6, 1198-1206 (1997).

${ }^{27}$ C. J. Chu, M. P. D’Evelyn, R. H. Hauge, and J. L. Margrave, J. Appl. Phys. 70, 1695-1705 (1991).

${ }^{28}$ M. P. D’Evelyn, C. J. Chu, R. H. Hauge, and J. L. Margrave, J. Appl. Phys. 71, 1528-1530 (1992).

${ }^{29}$ C. E. Johnson, W. A. Weimer, and F. M. Cerio, J. Mater. Res. 7, 14271431 (1992).

${ }^{30}$ M. H. Loh and M. A. Cappelli, Appl. Phys. Lett. 70, 1052-1054 (1997).

${ }^{31}$ D. N. Belton and S. J. Harris, J. Chem. Phys. 96, 2371-2377 (1992).

${ }^{32}$ J. E. Butler and R. L. Woodin, Philos. Trans. R. Soc. London, Ser. A 342, 209-224 (1993).
${ }^{33}$ M. Zhu, R. H. Hauge, J. L. Margrave, and M. P. D'Evelyn, in Evolution of Thin Film Microstructure, edited by H. A. Atwater, Mater. Res. Soc. Symp. Proc. 280 (MRS, Pittsburgh, 1993), pp. 683-688.

${ }^{34}$ S. J. Harris and D. N. Belton, Jpn. J. Appl. Phys., Part I 30, 2615-2618 (1991).

${ }^{35}$ S. Skokov, B. Weiner, and M. Frenklach, J. Phys. Chem. 99, 5616-5625 (1995).

${ }^{36} \mathrm{~S}$. Skokov, M. Frenklach, and B. Weiner, in Proceedings of the 4th International Symposium on Diamond Materials, edited by K. V. Ravi and J. P. Dismukes (Electrochemical Society, Pennigton, NJ, 1995), pp. 546-551.

${ }^{37}$ J. Warnatz, Combustion Chemistry, edited by W. C. Gardiner (Springer, Berlin, 1984).

${ }^{38}$ C. C. Battaile, D. J. Srolovitz, and J. E. Butler, J. Appl. Phys. 82, $6293-$ 6300 (1997).

${ }^{39}$ C. C. Battaile, D. J. Srolovitz, and J. E. Butler, in Thin Films: Surface and Morphology, edited by R. Cammarata, E. Chason, T. Einstein, and E. Williams, Mater. Res. Soc. Symp. Proc. 441 (MRS, Pittsburgh, 1997), pp. 509-514.

${ }^{40}$ C. C. Battaile, "Atomic-scale kinetic Monte Carlo simulations of diamond chemical vapor deposition," Ph.D. dissertation, The University of Michigan, 1998.

${ }^{41}$ A. B. Bortz, M. H. Kalos, and J. L. Lebowitz, J. Comput. Phys. 17, 10-18 (1975).

${ }^{42}$ D. T. Gillespie, J. Phys. Chem. 81, 2340-2361 (1977).

${ }^{43}$ B. J. Garrison, E. J. Dawnkaski, D. Srivastava, and D. W. Brenner, Science 255, 835-838 (1992).

${ }^{44}$ M. Frenklach and S. Skokov, J. Phys. Chem. B 101, 3025-3036 (1997).

${ }^{45}$ I. I. Oleinik, D. G. Pettifor, A. P. Sutton, and J. E. Butler, unpublished results.

${ }^{46}$ Cerius $^{2}$ Quantum Mechanics-Chemistry: ADF, DMol ${ }^{3}$, Gaussian, and MOPAC, Molecular Simulations Inc., 1998.

${ }^{47}$ A. D. Becke, J. Chem. Phys. 88, 2547-2553 (1988).

${ }^{48}$ J. P. Perdew and Y. Wang, Phys. Rev. B 45, 13244-13249 (1992).

${ }^{49}$ C. H. Xu, C. Z. Wang, C. T. Chan, and K. M. Ho, J. Phys.: Condens. Matter 4, 6047-6054 (1992).

${ }^{50}$ B. N. Davidson and W. E. Pickett, Phys. Rev. B 49, 11253-11267 (1994).

${ }^{51}$ A. P. Horsfield, P. D. Godwin, D. G. Pettifor, and A. P. Sutton, Phys. Rev. B 54, 15773-15775 (1996).

${ }^{52}$ Oxford Order N Package, Oxford University, 1996.

${ }^{53}$ M. Frenklach and C. S. Carmer, in Advances in Classical Trajectory Methods, Vol. IV: Molecular Dynamics of Clusters, Surfaces, Liquids, and Interfaces, edited by W. L. Hase (JAI Press, Stamford, CT, in press).

${ }^{54}$ I. I. Oleinik, D. G. Pettifor, A. P. Sutton, C. C. Battaile, D. J. Srolovitz, and J. E. Butler, in Multiscale Modeling of Materials, Mater. Res. Soc. Symp. Proc. (in press).

${ }^{55}$ S. Skokov, B. Weiner, and M. Frenklach, J. Phys. Chem. 98, 7073-7082 (1994).

${ }^{56}$ S. Skokov, B. Weiner, and M. Frenklach, J. Phys. Chem. 98, 8-11 (1994).

${ }^{57}$ C. T. Bowman, R. K. Hanson, D. F. Davidson, W. C. Gardiner, V. Lissianski, G. P. Smith, D. M. Golden, M. Frenklach, and M. Goldenberg, "'GRI-Mech,"' http://www.me.berkeley.edu/gri_mech/(1996).

${ }^{58}$ D. J. Chadi, Phys. Rev. Lett. 59, 1691-1694 (1987).

${ }^{59}$ C. C. Battaile, D. J. Srolovitz, and J. E. Butler, unpublished results.

${ }^{60}$ E. Kondoh, T. Ohta, T. Mitomo, and K. Ohtsuka, J. Appl. Phys. 73, 3041-3046 (1993).

${ }^{61}$ Z. Sun, Z. Zheng, N. Xu, and X. Zhang, Mater. Sci. Eng., B 25, 47-52 (1994).

${ }^{62}$ S. J. Harris and L. R. Martin, J. Mater. Res. 5, 2313-2319 (1990).

${ }^{63}$ S. S. Lee, D. W. Minsek, D. J. Vestyck, and P. Chen, Science 263, 15961598 (1994).

${ }^{64}$ R. E. Rawles, R. Gat, W. G. Morris, and M. P. D'Evelyn, in Diamond for Electronic Applications, edited by D. L. Dreifus, A. Collins, T. Humphreys, K. Das, and P. E. Pehrsson, Mater. Res. Soc. Symp. Proc. 416 (MRS, Pittsburgh, 1996), pp. 299-304.

${ }^{65}$ N. C. Burton, J. E. Butler, A. R. Lang, and J. W. Steeds, Proc. R. Soc. London, Ser. A 449, 555-566 (1995).

${ }^{66}$ K. Hayashi, S. Yamanaka, H. Okushi, and K. Kajimura, Appl. Phys. Lett. 68, 1220-1222 (1996). 